\title{
Tasa de reinfección por Helicobacter pylori en una cohorte de pacientes colombianos tratados exitosamente con seguimiento superior a 2 años
}

\author{
Helicobacter Pylori Reinfection Rate after More Than Two Years of Follow-up \\ in a Cohort of Successfully Treated Colombian Patients
}

William Otero Regino, MD, ${ }^{1}$ Alba Alicia Trespalacios Rangel, Bact. MSc, PhD, ${ }^{2}$ Marcela Mercado Reyes, Bact MSc. ${ }^{3}$

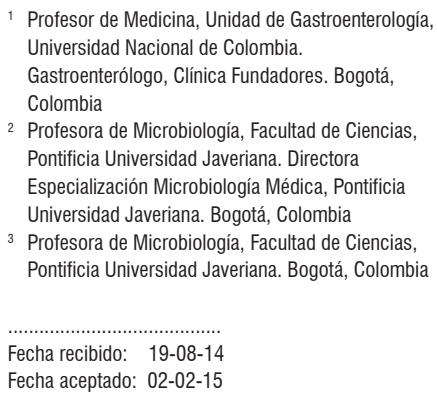

Profesor de Medicina, Unidad de Gastroenterología, Universidad Nacional de Colombia.

Gastroenterólogo, Clínica Fundadores. Bogotá,

Colombia

2 Profesora de Microbiología, Facultad de Ciencias, Pontificia Universidad Javeriana. Directora Especialización Microbiología Médica, Pontificia Universidad Javeriana. Bogotá, Colombia

3 Profesora de Microbiología, Facultad de Ciencias, Pontificia Universidad Javeriana. Bogotá, Colombia

\begin{abstract}
Resumen
El Helicobacter pylori (H. pylori) infecta por lo menos a la mitad de la población mundial, aunque la prevalencia es más alta en países subdesarrollados. La tasa de reinfección es variable en las diferentes regiones y puede incluir recrudescencia de la infección o verdadera recurrencia. Hasta el momento hay pocos estudios en Colombia que hayan investigado la recurrencia de la infección y ninguno se ha realizado en Bogotá, Colombia. Objetivo: determinar la tasa de recurrencia de $H$. pylori en pacientes tratados eficazmente con 3 terapias triples diferentes. Materiales y métodos: estudio observacional analítico anidado en una cohorte de 180 pacientes a la que se le erradicó exitosamente $H$. pylori durante 2008-2009. La erradicación se verificó con test respiratorio con urea marcada y el tiempo promedio de seguimiento fue 43,7 meses (rango 31-56 meses). La recurrencia se investigó con la prueba de antígenos fecales monoclonales ImmunoCard STAT ${ }^{\circledR}$ HpSA (Meridian Bioscience Inc.). Resultados: se siguieron 86 pacientes y el porcentaje total de reinfección por H. pylori fue de 5,8\% (5/86) y la tasa anual de reinfección fue de 1,59\% (5/313,4 pacientes año x 100). El primer caso de reinfección se presentó a los 32 meses y los demás ocurrieron a los 37, 42, 44 y 56 meses de seguimiento. La tasa de reinfección calculada fue 1,8\%/año después de 2 años de seguimiento. Conclusiones: la tasa de reinfección de $H$. pylori en Bogotá es baja e inferior a la previamente informada para otras regiones de Colombia.
\end{abstract}

\section{Palabras clave}

Reinfección, Helicobacter, recrudescencia.

\begin{abstract}
Helicobacter pylori $(\mathrm{H}$. pylori) infects at least half the world's population although its prevalence is higher in developing countries. The reinfection rate varies from region to region and may include recrudescence of infection or true recurrence. So far there are few studies that have investigated recurrence of infections in Colombia, and none had been done in Bogota, Colombia. Objective: The objective of this study was to determine the rate of recurrence of $\mathrm{H}$. pylori in patients who had been treated effectively with three different triple therapies. Materials and Methods: This was an observational study nested in a cohort of 180 patients in whom H. pylori had been successfully eradicated during 2008 and 2009. Eradication was verified with the labeled urea breath test. The average follow-up time was 43.7 months (range: $31-56$ months). Recurrence was investigated with monoclonal stool antigen tests (ImmunoCard STAT®, HpSA (Meridian Bioscience Inc.)). Results: A total of 86 patients were monitored during the follow-up period. Out of this group the reinfection rate was $5.8 \%(5 / 86)$. The annual reinfection rate was $1.59 \%$ (5/313.4 patient-years $\times 100)$. The first case of reinfection was presented at 32 months, and the other cases occurred at 37, 42, 44 and 56 month of followup. The reinfection rate was 1.8\%/year calculated after two years of monitoring. Conclusions: The $\mathrm{H}$. pylori reinfection rate in Bogotá is low and is less than that previously reported for other regions of Colombia.
\end{abstract}

\section{Keywords}

Reinfection, Helicobacter, recrudescence. 


\section{INTRODUCCIÓN}

El Helicobacter pylori (H. pylori) infecta aproximadamente al $50 \%$ de la población mundial $(1,2)$, pero su prevalencia varía significativamente entre los diferentes países, niveles sociales, edades y razas (3-5). Generalmente es más baja en países desarrollados que en países en vías de desarrollo, en los cuales puede ser del 80\% (6). Este microorganismo es el principal agente causal de gastritis crónica, úlceras pépticas y de adenocarcinoma del estómago (ACG) (7-10). Su erradicación cura o reduce notablemente las recurrencias de las úlceras pépticas (11), cura del $60 \%$ al $80 \%$ de los linfomas MALT gástricos de bajo grado en estadio temprano (Lugano I/II) $(12,13)$, y puede disminuir el riesgo de ACG $(14,15)$ y su recurrencia posresección endoscópica (16). Su erradicación es de bajo costo, eficaz en pacientes con dispepsia funcional (17) y mejora el conteo de plaquetas en el 50\% de aquellos con trombocitopenia inmune (18). Al tener en cuenta los beneficios de la erradicación de este microbio se le da importancia a la determinación de la tasa de reinfección, ya que al reaparecer el paciente volverá a tener gastritis así como recurrencias de úlceras pépticas $(19,20)$. El impacto es aún mayor en países como Colombia, con prevalencia de la infección (77,2\%-83\%) (21-23) y alta incidencia de ACG (17,4-48,2/100000hb) (24).

La reinfección es una forma de recurrencia y se define como la reaparición de $H$. pylori después de una prueba negativa realizada luego de 4 semanas de haber terminado un tratamiento de erradicación (20). La recurrencia puede ser secundaria a "recrudescencia" o a una verdadera reinfección. La recrudescencia es la situación en la cual la cepa de $H$. pylori, presente antes del tratamiento, es temporalmente suprimida por la medicación con antibióticos e indetectable en las pruebas de seguimiento realizadas 4 semanas postratamiento, pero se manifiesta nuevamente cuando el microorganismo vuelve a replicarse $(20,25)$; esta definición implica que la bacteria identificada (por técnicas moleculares) es la misma que había antes del tratamiento. En la reinfección, el $H$. pylori detectado es molecularmente diferente o genéticamente idéntico al encontrado en una fuente original común después de ser erradicado (20).

A nivel mundial las tasas de recurrencia son variables, sin embargo, se presentan más frecuentemente en el mundo subdesarrollado. En una revisión sistemática en 2005, Gisbert encontró una recurrencia anual de 3\% por año (20) y consideró que probablemente era secundaria a recrudescencia y no a verdadera reinfección, ya que la mayoría declinaba con el tiempo. Diversos estudios han demostrado que las reinfecciones son infrecuentes después del primer año de tratamiento y que la mayoría de las recurrencias se deben a recrudescencias; sin embargo, la utilización de métodos inadecuados tanto para verificar la erradicación de la infección como para realizar seguimiento dificultan el conocimiento de la verdadera tasa de reinfección (25). Un metaanálisis reciente (26) encontró que en países desarrollados la recurrencia anual en promedio es de $2,7 \%$ y en los países subdesarrollados del 13\% o más. En Colombia hace más de 15 años se encontraron tasas de recurrencia de $18 \%$ a $20 \%$ al año $(27,28)$; no obstante, la evaluación se hizo durante el primer año y un poco después utilizando el test de ureasa rápida e histología con hematoxilina y eosina, sin coloraciones especiales, con lo cual quedan dudas por la probabilidad de resultados inexactos (20). Sin embargo, en un estudio latinoamericano multinacional reciente que utilizó el test respiratorio de urea, la tasa de recurrencia para Colombia fue de $18,1 \%$ (los participantes eran de Túquerres, Nariño) y el promedio para América de 11,5\% (29). Teniendo en cuenta que los estudios sobre reinfección en Colombia han dado altas tasas con métodos inexactos y tasas similares con el test respiratorio de urea pero en zonas rurales, se decidió realizar un estudio riguroso para determinar la tasa de reinfección en Bogotá en una cohorte de pacientes tratados con terapias triples y seguidas durante 2-5 años.

\section{MATERIALES Y MÉTODOS}

Estudio de cohorte en pacientes curados de la infección en la Clínica Fundadores y en la Facultad de Ciencias de la Pontificia Universidad Javeriana.

\section{Población y muestra}

Durante los años 2008 a 2009 fue desarrollado el experimento clínico, aleatorizado, ciego simple de tratamiento de $H$. pylori con el uso de 3 terapias triples diferentes, y que incluyó 80 pacientes en cada rama. Para este experimento clínico se identificó el $H$. pylori mediante prueba de ureasa rápida o histología con hematoxilina y eosina, y adicionalmente coloración de Giemsa o por cultivo. En ese estudio, los pacientes recibieron 1 de 3 tipos de terapias triples durante 10 días: 1. esomeprazol + levofloxacina + amoxicilina (ELA); 2. esomeprazol + levofloxacina + claritromicina (ELC); y 3. esomeprazol + claritromicina + amoxicilina (ECA). Luego de 8 semanas de terminado el tratamiento se les verificó erradicación exitosa del $H$. pylori con la prueba de aliento con urea $13 \mathrm{C}$ (PAU), como ha sido descrito previamente (19). Una vez determinado el éxito terapéutico, los pacientes fueron invitados a ser parte de una cohorte de seguimiento para evaluar reinfección y después de terminado el tratamiento, los pacientes incluidos en el seguimiento por al menos 1 año fueron llamados vía telefónica para realizarse un test de antígenos fecales con el fin de evaluar reinfección. Se utilizó la prueba de antígenos 
fecales (ImmunoCard STAT HpSA Meridian Bioscience Inc.) considerada por el Consenso de Maastricht como útil cuando no se dispone del test respiratorio de urea (15). Esa prueba tiene sensibilidad del 95,4\%, (IC 95\% 86\%-100\%) y especificidad de $100 \%$.

\section{Objetivos generales}

Determinar la tasa de reinfección anual por $H$. pylori en individuos tratados exitosamente con 3 triples terapias diferentes.

\section{Objetivos específicos}

1. Determinar la tasa de reinfección de acuerdo al tiempo de seguimiento de los individuos.

2. Identificar posibles factores de riesgo asociados con la reinfección

\section{Criterios de exclusión}

Pacientes con diagnóstico o sospecha de cáncer gástrico, mujeres en embarazo o lactancia, antecedente de cirugía gástrica previa, utilización actual de antibióticos o medicamentos antisecretores o bismuto por lo menos 1 mes antes de realizar la prueba de antígenos fecales.

\section{Variables}

Variable dependiente: tasa de reinfección: cualquier resultado positivo de antígenos fecales durante el período de seguimiento.

Variables independientes: variables sociodemográficas (edad, sexo, estrato socioeconómico, grado de escolaridad, ocupación, etc.), variables de antecedentes familiares (familiares con diagnóstico de infección por H. pylori, antecedentes de enfermedad gastroduodenal, etc.), tipo de tratamiento administrado, tiempo de seguimiento antes de la reinfección.

\section{Procedimiento}

Reclutamiento de pacientes: los sujetos que cumplían con los criterios de inclusión en el estudio fueron invitados a participar, se les explicó de manera detallada el objeto de estudio y se les solicitó firmar un consentimiento informado. Las variables de estudio fueron consignadas en un formato precodificado. Una vez aprobada la participación, se les realizó la prueba de antígenos fecales con el fin de determinar la tasa de reinfección.
Procedimiento para la prueba de antígenos fecales: para esta prueba se le indicó al paciente que debía tomar una muestra de materia fecal y no consumir medicamentos inhibidores de la bomba de protones o antiácidos por lo menos 2 semanas previas al examen. Una vez recolectada la muestra fue entregada al laboratorio para su análisis y fue procesada el mismo día de su recolección. Para determinar la presencia de antígenos de $H$. pylori en las muestras de materia fecal se utilizó la prueba ImmunoCard STAT ${ }^{\circ}$ HpSA (Meridian Bioscience Inc.) siguiendo el protocolo recomendado por el fabricante y consignado en el catálogo 750720. Ésta se fundamenta en un inmunoensayo cualitativo y rápido para la detección in vitro de antígenos de $H$. pylori en materia fecal humana; para su procesamiento se dispensa una muestra diluida de materia fecal del paciente en el puerto de muestra del dispositivo de ensayo y, al cabo de 5 minutos de incubación a temperatura ambiente, la aparición de una línea de color rosa-rojo en la ventana contigua a la letra " $T$ " indica un resultado positivo. Tanto el protocolo de investigación como el consentimiento informado fueron aprobados por el Comité de Ética de la institución en donde se realizó el estudio.

Recolección, procesamiento y análisis de resultados: se creó una base de datos en el programa SPSS $20^{\circ}$. Para el análisis de la variable dependiente se calculó la tasa de reinfección persona-tiempo en meses y la frecuencia de reinfección durante el seguimiento con curvas de sobrevida usando Kaplan-Meier. Para evaluar la asociación entre la reinfección con los posibles factores de riesgo se realizó un análisis univariado y se calcularon riesgos relativos con sus respectivos intervalos de confianza del $95 \%$. Todos los resultados se consideraron estadísticamente significativos cuando la significancia fue de $<0,05$.

\section{RESULTADOS}

De los 180 pacientes elegibles, finalmente 86 fueron incluidos en este estudio; de estos, 60 no participaron porque al momento de citarlos para la prueba de antígenos fecales estaban recibiendo antibióticos (20 pacientes), o tratamiento con inhibidores de bomba de protones por reflujo gastroesofágico (30 pacientes), o estaban hospitalizados por patologías pulmonares o por cirugía (10 pacientes); además, 34 pacientes no entraron al estudio porque no desearon participar. Con respecto al tratamiento de erradicación recibido, 36 pacientes $(41,9 \%)$ recibieron terapia ELA, $26(30,2 \%)$ terapia ELC y $24(27,9 \%)$ terapia ECA. La media de edad del grupo fue de $53 \pm 11$ años (media \pm DS; rango, 26-74 años); de éstos, 56 (65\%) fueron mujeres y 30 (36\%) hombres. El diagnóstico clínico principal fue gastritis crónica en 
50 pacientes $(59,5 \%)$, seguido de dispepsia funcional B1a o B1b en 22 (26\%) y úlcera péptica en 2 (2,4\%). En la figura 1 se muestra el diseño del estudio. La información sociodemográfica y clínica se muestra en la tabla 1.

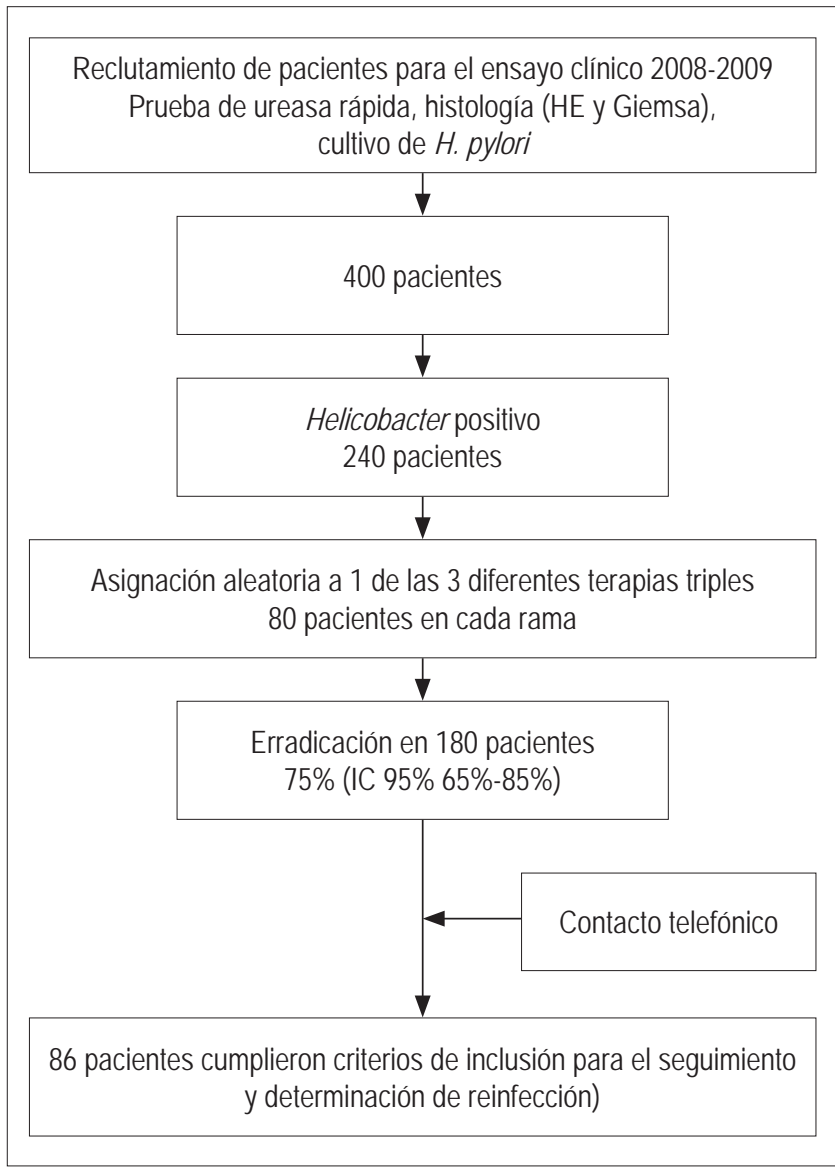

Figura 1. Diseño del estudio.

\section{Seguimiento y tasa de reinfección}

La media de duración del tiempo de seguimiento en el grupo de 86 pacientes fue de 43,7 meses (rango 31-56 meses). El porcentaje total de reinfección por $H$. pylori fue de 5,8\% (5/86); de estos casos, $3 / 5$ estuvieron en el grupo de pacientes que recibieron como terapia triple ELA, 2/5 tuvieron reinfección en el grupo que recibió la terapia ECA y no se presentó ningún caso de reinfección en el grupo de pacientes que recibieron la terapia ELC. La tasa anual de reinfección fue de 1,59\% (5/313,4 pacientes año x 100) y la distribución según el tiempo de seguimiento se observa en la tabla 2. En la curva de ocurrencia de reinfección se encontró que el primer caso se presentó a los 32 meses y posteriormente aparecen casos a los 37, 42, 44 y 56 meses de seguimiento, como se muestra en la figura 2 . No se observaron asociaciones significativas con los factores de riesgo analizados para reinfección.
Tabla 1. Características de los pacientes.

\begin{tabular}{|c|c|c|c|}
\hline Variable & Total & $\begin{array}{c}\text { Grupo } \\
\text { reinfectado }\end{array}$ & $\begin{array}{c}\text { Grupo } \\
\text { sin infección }\end{array}$ \\
\hline $\begin{array}{l}\text { Género } \\
\text { Femenino } \\
\text { Masculino }\end{array}$ & $\begin{array}{c}n=86 \\
56(65 \%) \\
30(36 \%)\end{array}$ & $\begin{array}{c}n=5 \\
3(60 \%) \\
2(40 \%)\end{array}$ & $\begin{array}{c}n=81 \\
53(65 \%) \\
28(35 \%)\end{array}$ \\
\hline $\begin{array}{l}\text { Edad (años) } \\
\text { (media } \pm \text { DS) } \\
20-29 \\
30-39 \\
40-49 \\
50-59 \\
60-69 \\
70-79\end{array}$ & $\begin{array}{c}n=85 \\
(53 \pm 11) \\
5(5,9) \\
5(5,9) \\
16(18,8) \\
33(38,8) \\
22(25,9) \\
4(4,7)\end{array}$ & $\begin{array}{c}n=5 \\
(54 \pm 10,2) \\
2(40) \\
0(0) \\
2(40) \\
0(0) \\
0(0) \\
1(20)\end{array}$ & $\begin{array}{c}n=80 \\
(44 \pm 18) \\
3(3,8) \\
5(6,3) \\
14(17,5) \\
33(41,3) \\
22(27,5) \\
3(3,8)\end{array}$ \\
\hline $\begin{array}{l}\text { Estado de fumador } \\
\text { Sí } \\
\text { No }\end{array}$ & $\begin{array}{c}n=85 \\
9(10,6) \\
76(89,4)\end{array}$ & $\begin{array}{c}n=5 \\
0(0) \\
5(100)\end{array}$ & $\begin{array}{c}n=80 \\
9(11,3) \\
71(88,8)\end{array}$ \\
\hline $\begin{array}{l}\text { Ingesta de alcohol } \\
\text { Sí } \\
\text { No }\end{array}$ & $\begin{array}{c}n=85 \\
14(16,5) \\
71(83,5)\end{array}$ & $\begin{array}{c}n=5 \\
0(0) \\
5(100)\end{array}$ & $\begin{array}{c}n=80 \\
14(17,5) \\
66(82,5)\end{array}$ \\
\hline $\begin{array}{l}\text { Frecuencia de ingesta } \\
\text { Una vez por semana } \\
\text { Dos o más veces por } \\
\text { semana }\end{array}$ & $\begin{array}{c}n=14 \\
12(85,7) \\
2(14,3)\end{array}$ & & $\begin{array}{c}n=14 \\
12(85,7) \\
2(14,3)\end{array}$ \\
\hline $\begin{array}{l}\text { Ingresos mensuales por } \\
\text { familia } \\
\text { Hasta } 3 \mathrm{SML} \\
\text { Más de } 3 \mathrm{SML}\end{array}$ & $\begin{array}{l}n=82 \\
22(26,8) \\
60(73,2)\end{array}$ & $\mathrm{n}=51(20)$ & $\begin{array}{c}n=77 \\
21(27,3) \\
56(72,7)\end{array}$ \\
\hline $\begin{array}{l}\text { Nivel de escolaridad } \\
\text { Alto (grado universitario } \\
\text { /posgrado) }\end{array}$ & $\begin{array}{l}n=84 \\
68(81)\end{array}$ & $\begin{array}{l}n=5 \\
3(60)\end{array}$ & $\begin{array}{c}n=79 \\
65(85,3)\end{array}$ \\
\hline $\begin{array}{l}\text { Medio (grado } \\
\text { de secundaria o } \\
\text { universidad incompleta) }\end{array}$ & $12(14,3)$ & $2(40)$ & $10(12,7)$ \\
\hline $\begin{array}{l}\text { Bajo (primaria y/o } \\
\text { secundaria incompleta) }\end{array}$ & $4(4,7)$ & $0(0)$ & $4(5,1)$ \\
\hline $\begin{array}{l}\text { Diagnóstico clínico } \\
\text { Úlcera péptica } \\
\text { Dispepsia funcional B1a } \\
\text { o B1b }\end{array}$ & $\begin{array}{l}n=84 \\
2(2,4) \\
22(26)\end{array}$ & $\begin{array}{l}n=5 \\
2(40) \\
2(40)\end{array}$ & $\begin{array}{c}n=79 \\
2(2,5) \\
20(25,3)\end{array}$ \\
\hline $\begin{array}{l}\text { Gastritis crónica } \\
\text { Ninguno } \\
\text { Otros }\end{array}$ & $\begin{array}{c}50(59,5) \\
4(4,8) \\
6(7,1)\end{array}$ & $\begin{array}{l}1(20) \\
0(0) \\
0(0)\end{array}$ & $\begin{array}{c}48(60,8) \\
3(3,8) \\
6(7,6)\end{array}$ \\
\hline $\begin{array}{l}\text { Tipo de tratamiento de } \\
\text { erradicación }\end{array}$ & $n=86$ & $n=5$ & $n=81$ \\
\hline ELA & $36(41,9)$ & $3(60)$ & $33(40,7)$ \\
\hline ELC & $26(30,2)$ & $0(0)$ & $26(32,1)$ \\
\hline ECA & $24(27,9)$ & $2(40)$ & $22(27,2)$ \\
\hline
\end{tabular}

\section{DISCUSIÓN}

En el presente estudio, con un seguimiento de 2 a 5 años, el riesgo acumulativo de recurrencia de la infección fue de $5,8 \%$ y la tasa de positividad de $H$. pylori fue de $1,8 \% /$ año después de 2 años de seguimiento. No hubo diferencia estadísticamente significativa entre la tasa de reinfección y 
Tabla 2. Tiempo de seguimiento de los pacientes.

\begin{tabular}{lcccc}
\hline Periodo de seguimiento & Número de pacientes & Número de pacientes reinfectados & Pacientes-año & Tasa de reinfección anual (\%) \\
\hline $\begin{array}{l}25-36 \text { meses } \\
\text { 2 a } 3 \text { años }\end{array}$ & 20 & 1 & 55,5 & 1,8 \\
37-48 meses & 40 & 3 & 141,4 & 2,12 \\
3 a 4 años & 26 & 1 & 116,5 & 0,86 \\
$\begin{array}{l}49-60 \text { meses } \\
4 \text { a } 5 \text { años }\end{array}$ & 86 & 5 & 313,4 & 1,59 \\
\hline Total & & & \\
\hline
\end{tabular}

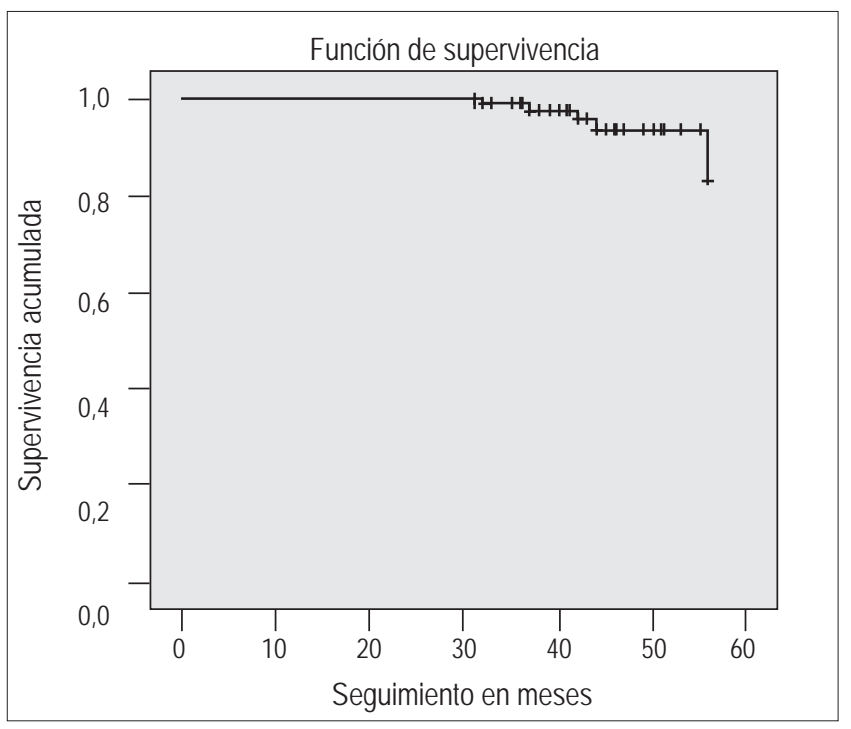

Figura 2. Curva de Kaplan-Meier para reinfección por Helicobacter pylori.

el tipo de terapia recibida, aunque por el bajo número de reinfectados no se puede determinar con exactitud esta relación. La aparición tardía del microorganismo está a favor de una reinfección y no de recrudescencia ya que ésta última aparece durante el primer año o inmediatamente después del mismo $(20,25)$. Respalda aún más la probabilidad de reinfección, la disminución de la tasa de recurrencia durante el $4 .^{\circ}$ y el $5 .^{\circ}$ año de seguimiento durante los cuales fue de $0,86 \%$. Los 5 pacientes reinfectados fueron sometidos a endoscopia de control y se les tomaron biopsias para cultivo y caracterización molecular del $H$. pylori recuperado para determinar si son diferentes a las cepas encontradas antes del tratamiento de erradicación, sin embargo, esta información no estuvo disponible al momento de escribir este artículo. La tasa de recurrencia encontrada en este estudio contrasta con la alta tasa de recurrencia de 18,2\% al año encontrada recientemente en la población rural de Túquerres, Nariño, dentro del estudio latinoamericano de recurrencia de la infección por $H$. pylori (29), y también es inferior al $11,5 \%$ en promedio de los países latinoamerica- nos que hicieron parte de ese estudio. Existe la posibilidad que esas tasas más altas sean secundarias a una mezcla de recrudescencia y de reinfección, teniendo en cuenta que el microorganismo fue identificado inmediatamente después del primer año $(20,25,30)$.

Los resultados del presente estudio también son diferentes a los encontrados en el más grande estudio de seguimiento en Latinoamérica con una duración de 6 años (31), donde la tasa de reinfección fue de $5,4 \%$ por año y se determinó por histología (31). Los pacientes incluidos en ese seguimiento también hicieron parte de un ensayo clínico realizado 10 años antes (32) en una población rural, en el cual la erradicación inicial de H. pylori fue realizada con test respiratorio de urea; sin embargo, no se precisa cuánto tiempo después del tratamiento y este aspecto es muy importante para descartar la posibilidad de resultados iniciales falsos negativos. Se desconoce la razón de esta discrepancia en los resultados aunque no se descartan factores de contexto como la población pequeña, rural, con diferentes condiciones higiénicas y sanitarias, y que está expuesta a una mayor probabilidad de producir contaminación del medio ambiente con $H$. pylori a través del agua, vegetables no completamente cocinados y otros factores medioambientales, los cuales son considerados como muy importantes en la reexposición a la infección por H. pylori, como se ha demostrado en países subdesarrollados que tienen alta prevalencia de la infección $(33,34)$. Otros factores que también pueden favorecer la reinfección en ese tipo de poblaciones son la transmisión intrafamiliar (verdadera reinfección), así como la transmisión a través de endoscopios y pinzas de biopsias reprocesados manualmente, los cuales la pueden transmitir en 1\%-3\% de las endoscopias, incluso si los equipos son lavados con etanol al $70 \%$ o glutaraldehído al $2 \%$ (35).

Los pacientes del estudio inicial fueron sometidos a varias endoscopias como parte del proceso de determinación de la evolución de lesiones precursoras de cáncer gástrico (32). En nuestro trabajo, la prevalencia de la infección pretratamiento fue de 59,5\% (IC 95\% 54,7\%-64,4\%) y los pacientes que hicieron parte del mismo eran de una 
ciudad capital de estrato 3-5; además, el estudio se realizó 20 años después del trabajo realizado en Túquerres, cuya prevalencia de la infección 20 años después (2013) es del $83 \%$ (22), mucho más alta que el 59,5\% encontrado en este estudio. Se cree que los factores mencionados podrían justificar la discrepancia de los diferentes resultados de estos dos estudios. Con las características de diseño del presente estudio y sus resultados consideramos que, por lo menos en Bogotá, en la actualidad la prevalencia de H. pylori es inferior a la informada previamente para otras regiones del país (59,5\% versus $77 \%-83 \%$ ) pero más alta que la encontrada en países industrializados, que es del 10\% al 30\% (36); además, la tasa de reinfección es baja, similar a la encontrada muy recientemente en Brasil que fue de 1,8\% al año con seguimiento a 5 años (37), y parecida también a la de otro país subdesarrollado como Marruecos, en el cual se encontró que fue del $0,8 \%$ al año (36). En otros países del mundo subdesarrollado las tasas de reinfección varían desde 1,1\% a los 2 años en China (38) a 73\% a los 8 meses en Perú (39).

\section{CONCLUSIONES}

Los resultados del presente estudio demuestran, en primer lugar, que la prevalencia de la infección en Bogotá determinada en un grupo de pacientes con dispepsia o síntomas de enfermedad por reflujo gastroesofágico es del $59 \%$, la cual es inferior a la encontrada previamente. En segundo lugar, la tasa de reinfección es baja (1,59\%/año), y por este dato se justifica optimizar el manejo de la infección en las indicaciones recomendadas (15). Con esta nueva información, el paciente y el médico podrían estar más tranquilos hacia el futuro sin temor a que la reinfección sea "inevitable", como frecuentemente se piensa en muchos sitios. Consideramos importante continuar con este tipo de estudios en otras regiones de Colombia ya que la epidemiología de $H$. pylori puede variar en diferentes sitios dentro de un mismo país.

\section{Conflicto de intereses}

Ninguno. Los costos del presente estudio fueron asumidos en su totalidad por los investigadores.

\section{REFERENCIAS}

1. Dunn BE, Cohen H., Blaser MJ. Helicobacter pylori. Clin Microbiol Rev. 1997; 10:720-41.

2. Suerbaum S, Michetti P. Helicobacter pylori infection. N Engl J Med 2002;347:1175-86.

3. Grad YH, Lipsitch M., Aiello AE. Secular trends in Helicobacter pylori seroprevalence in adults in the United States: Evidence for sustained race/ethnic disparities. Am J Epidemiol 2012;175:54-9.
4. Epplein M, Signorello LB, Zheng W, Peek RM, Michel A, Williams SM, et al. Race, African ancestry, and Helicobacter pylori infection in a low-income United States population. Cancer Epidemiol Biomarkers Prev 2011;20:826-34.

5. Jafar S, Jalil A, Soheila N, Sirous S. Prevalence of Helicobacter pylori infection in children, a population-based cross-sectional Study in West Iran. Iran J Pediatr 2013;23:13-8.

6. Sanders MK, Peura DA. Helicobacter pylori-associated diseases. Curr Gastroenterol Rep 2002;4:448-54.

7. NIH Consensus Conference. Helicobacter pylori in peptic ulcer disease. NIH Consensus Develpment Panel on Helicobacter pylori in Peptic Ulcer Disease. JAMA 1994;272:65-69.

8. McKoll KE. Clinical practice. Helicobacter pylori infection. $\mathrm{N}$ Engl J Med 2010;362:1597-1604.

9. Mbulaiteye SM, Hisada M, El-Omar EM. Helicobacter pylori associated global gastric cancer burden. Front Biosci 2009; 14:1490-1504

10. Piazuelo MB, Epplein M, Correa P. Gastric Cancer: An infectious disease. Infect Dis Clin N Am 2010;24:853-69.

11. Hopkins RJ, Girardi LS, Turney EA. Relationship between Helicobacter pylori eradication and reduced duodenal and gastric peptic ulcer recurrence: A review. Gastroenterology 1996; 110:1244-52.

12. Chen LT, Lin JT, Tai JJ. Long-term results of anti-Helicobacter pylori Therapy in early-stage gastric high grade transformed MALT lymphoma. J Natl Cancer Inst 2005;97:1345-53.

13. Sthatis A, Chini C, Bertoni F. Long-term outcome following Helicobacter pylori eradication in a retrospective study of 105 patients with localized gastric marginal zone B-cell lymphoma of MALT type. Ann Oncol 2009;20:1086-93.

14. Wong BC, Lam SK, Wong WM. Helicobacter pylori eradication to prevent gastric cancer in a high risk region in China: A randomizes controlled trial. JAMA 2004;291:187-94.

15. Malfertheiner P, Megraud F, O'Morain CA. Management of Helicobacter pylori infection. The Masstricht IV/Florence Consensus Report. Gut 2012;61:646-64.

16. Fukase K, Kato M, Kikuchi S. Effect of eradication of Helicobacter pylori on incidence of metachronous gastric carcinom after endoscopic resection of early gastric cancer: An open-label randomized controlled trial. Lancet 2008;372:392-7.

17. Moayyedi P. Dyspepsia. Curr Opin Gastroenterol 2012;28:602-7.

18. Stasi R, Sarpatwari A, Segal JB, Osborn J, Evangelista ML, Cooper $\mathrm{N}$, et al. Effects of eradication of Helicobacter pylori infection in patients with immune thrombocytopenic purpura: A systematic review. Blood 2009; 113:1231-40.

19. Xia HH, Talley NJ, Keane CT. Recurrence of Helicobacter pylori infection after successful eradication: Nature, possible causes and potential preventive strategies. Dig Dis Sci 1997;42:1821-24.

20. Gisbert J. The recurrence of Helicobacter pylori infection: Incidence and variables influencing it. A critical review. Am J Gastroenterol 2005;100:2083-99. 
21. Campuzano-Maya G, Hoyos D, Calvo VD, Suárez OA, Lizcano D, Rojas CA. Prevalencia de la infección por Helicobacter pylori en médicos de Medellín, Colombia. Acta Gastroenterol Latinoam 2007;37:99-103.

22. Bravo LE, Cortés A, Carrascal E, Jaramillo R, García LS, Bravo PE, et al. Helicobacter pylori: patología y prevalencia en biopsias gástricas en Colombia. Col Med 2003;34:124-31.

23. Porras C, Nodora J, Sexton R, Ferrecccio C, Jiménez S, Domínguez RL, et al. Epidemiology of Helicobacter pylori infection in six Latin American countries (SWOG Trial S0701). Cancer Causes Control 2013;24:209-215.

24. http://globocan.iarc.fr Consultada el 7 de junio de 2013.

25. Zhang YY, Xia HHX, Zhuang ZH, Zhong J. Review article: "true" re-infection of Helicobacter pylori after successful eradication-worldwide annual rates, risk factors and clinical implications. Aliment Pharmacol Ther 2008;29:145-60.

26. Niv Y, Hazzai R. Helicobacter pylori recurrence in developed and developing countries: meta-analysis of 13C-urea breath test follow-up after eradication. Helicobacter 2008; 13:56-61.

27. Otero W, Sierra F, Gutiérrez O. Omeprazole plus Amoxicilin in H. pylori Erradication and Duodenal Ulcer. Reinfection and Ulcer recurrence in an area of high prevalence of infection. Gastroenterology 1995;108:A185.

28. Sierra F, Gutiérrez O, Molano B, Otero W. $H$ pylori: Reinfection rate after succesfull Eradication. Gastroenterology 1995;108:A21989.

29. Morgan DR, Torres J, Sexton R, Herrero R, Salazar-Martínez E, Greenberg ER, et al. Risk of recurrent Helicobacter pylori infection 1 year after initial eradication therapy in 7 Latin American Communities. JAMA 2013;309:578-86.

30. Rollan A, Giancaspero R, Fuster F, et al. The long-term reinfection rate and the course of duodenal ulcer disease after eradication of Helicobacter pylori in a developing country. Am J Gastroenterol 2000;95:50-56.

31. Mera R, Fotham ETH, Bravo LE, Bravo JC, Piazuelo MB, Camargo MC, et al. Long Term follow up of patients treated for Helicobacter pylori infection. Gut 2005;54:1536-40.

32. Correa P, Fontham ETH, Bravo JC, Bravo LE, Ruíz B, Zarama $\mathrm{G}$, et al. Chemoprevention of gastric displasia: Randomized trial of antioxidant supplements and Anti-Helicobacter pylori therapy. J Natl Cancer Inst 2000;92:1881-8.

33. Hulten K, Han SW, Enroth H. Helicobacter pylori in the drinking water in Peru. Gastroenterology 1996; 110:1035-6.

34. Hopkins RJ, Vial PA, Ferreccio C. Seroprevalence of Helicobacter pylori in Chile: Vegetables may serve as one route of transmission. J Infect Dis 1993;168:222-6.

35. Langenberg W, Raws EAJ, Oudbier JH. Patient to patient transmission of Campylobacter pylori infection by fiberoptic gastroduodenoscopy and biopsy. J infect Dis 1990;161:507-11.

36. Benajah DA, Lahbabi M, Alaoui S, El-Rhazi K, El Abkari E, Nejjari C, et al. Prevalence of Helicobacter pylori and its recurrence after successful eradication in a developing nation (Morocco). Clin Res Hepatol Gasroenterol 2013 early Rel April 6.

37. Silva FM, Navarro-Rodríguez T, Barbuti RC, Mattar R, Hashimoto CL, Eisig JN. Helicobacter pylori reinfection in Brazilian patients with peptic ulcer disease: A 5-year followup. Helicobacter 2010;15:46-52.

38. Mitchell HM, Hu P, Chi Y. A low rate of reinfection following effective therapy against Helicobacter pylori in a developing nation (China). Gastroenterology 1998;114:256-61.

39. Ramírez-Ramos A, Gilman HR, Leon-Barua R. Rapid recurrence of Helicobacter pylori infection in Peruvian patients after successful eradication. Clin Infect Dis 1997;25:1027-31. 